\title{
Pre-tibial myxedema: treatment with intralesional corticosteroid ${ }^{*}$
}

\author{
Luana Oliveira Ramos ${ }^{1}$ \\ Giuseppe Lemos Pertoti de Figueredo ${ }^{1}$ \\ Sandra Adolfina Reys Romero ${ }^{1}$
}

\author{
Paloma Corrêa Mattos ${ }^{1}$ \\ Alef Alioscha Andrade Maia ${ }^{1}$
}

DOI: http:/ / dx.doi.org/10.1590/abd1806-4841.20153651

\begin{abstract}
A bstract: The pretibial myxedema is a manifestation of Graves' disease characterized by accumulation of glycosaminoglycans in the reticular dermis. The dermopathy is self-limiting but in some cases may cause cosmetic and functional damage. Conventional treatment is use of topical steroids under occlusive dressing, however the intralesional application has shown good results. We present a case of pretibial myxedema treated with single injection of intralesional corticosteroid.
\end{abstract}

Keywords: Adrenal cortex hormones; Glucocorticoids; Graves disease; Myxedema

\section{INTRODUCTION}

Pretibial myxedema is a dermatological manifestation of Graves' disease, arising from a local autoimmune response of the connective tissue, probably caused by antibodies against receptors of thyroid-stimulating hormone (TSH). ${ }^{1}$ Its prevalence varies from 0.5 to $4 \%$ in patients with Graves' disease, and is more frequent (15\%) in those who carry severe ophthalmopathy. Even though the pathogenesis is uncertain, it is known that besides immunological effects, mechanical factors, such as trauma and prolonged standing posture, contribute to the formation of pretibial myxedema. $^{2}$

The diagnosis is clinical, based on characteristics of pretibial lesions, presence of Graves' ophthalmopathy and history of thyrotoxicosis. In doubtful cases, a biopsy may be performed. ${ }^{3}$

Histologically, the tissue presents wide spaces between collagen bundles under hematoxilin and eosin staining, showing accumulations of abundant mucopolysaccharides acid between collagen bundles of dermis through Alcian blue, coloidal iron or toluidine blue staining. It also presents normal or slightly elevated amount of fibroblasts; the presence of TSH receptors in activated fibroblasts seems to be the largest piece in the pathogenesis of dermatopathy. ${ }^{1,3}$

In general dermatopathy is self-limiting, however, advanced cases may present cosmetic and functional damage. Application of occlusive corticoid directly over the lesion has been reported as a successful therapeutic option in several studies, although the clinical response diminishes when lesions are larger in size. ${ }^{1}$ Recently, the use of intralesional corticoids has demonstrated positive results with shorter treatment time. ${ }^{4-6}$

\section{CASE REPORT}

Male patient, 23 years old, was referred to the dermatology service complaining about an asymptomatic lesion on the anterior face of lower third of right leg. At the dermatological examination, a plaque with a shiny surface, measuring three centimeters,

\footnotetext{
Received on 24.04.2014

Approved by the Advisory Board and accepted for publication on 03.06.2014

Work performed at Hospital Universitário Getúlio Vargas - Universidade Federal do Amazonas (HUGV-UFAM) - Manaus (AM), Brazil. Financial Support: None.

Conflict of Interest: None.

1 Universidade Federal do Amazonas (UFAM) - Manaus (AM), Brazil.

C2015 by Anais Brasileiros de Dermatologia
} 
with accentuation of follicular orifices similar to "orange peel" (Figure 1) was observed. Upon palpation, it presented a firm and non-depressible consistency. Clinical examination showed exophthalmos (Figure 2). Diagnosed with Graves' disease for three years, he underwent treatment with Tapazole $10 \mathrm{mg}$ and iodotherapy, and currently is using levothyroxine for thyroid hormone replacement. Histopathological samples obtained by incisional biopsy of lesions revealed accumulation of mucin in reticular dermis leading to separation of collagen bundles. Epidermis and papillary dermis without abnormalities (Figures 3 and 4). After diagnostic confirmation, the option was for intralesional infiltration with corticosteroid. Triamcinolone $20 \mathrm{mg} / \mathrm{mL}$ diluted with anesthetic (lidocaine hydrochloride $2 \%$ ) was used, reaching a concentration of

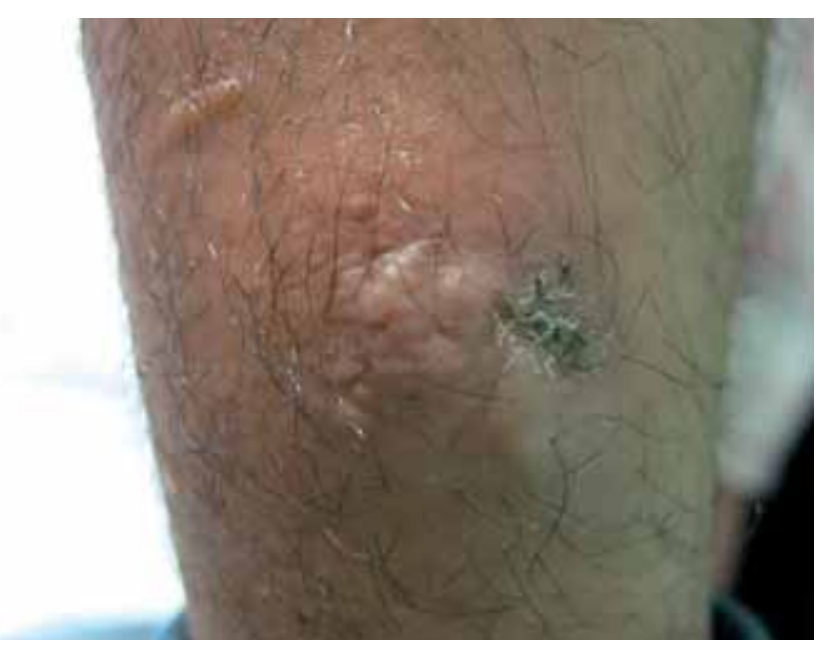

Figure 1: Plaque with "orange peel" surface on anterior face of right leg

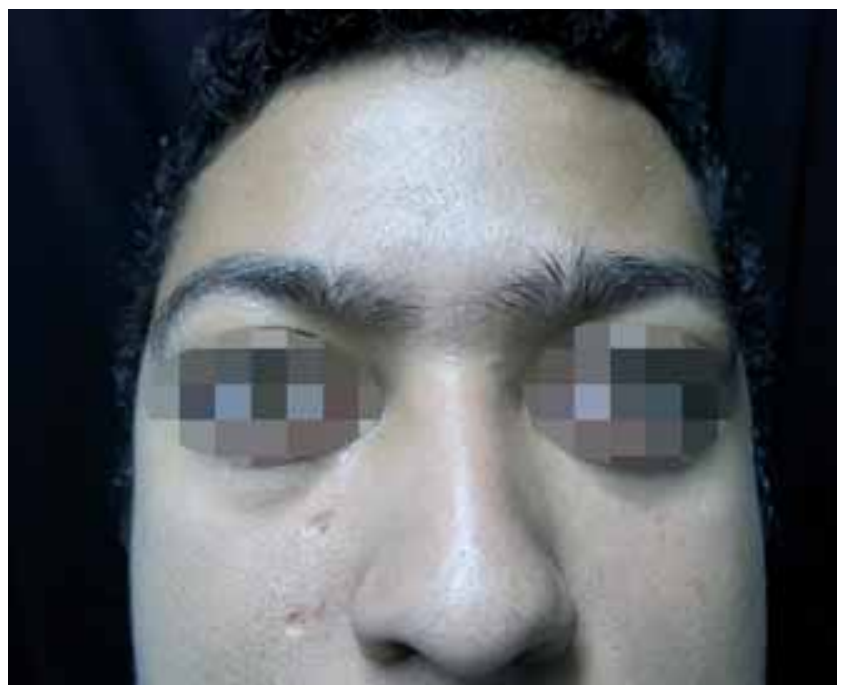

Figure 2: Exophthalmos
$5 \mathrm{mg} / \mathrm{mLin}$ a total volume of $1.5 \mathrm{~mL}$; the puncture was a single one, with a fan distribution pattern. The lesion remitted 30 days after this single application, resulting in a brownish macule (Figure 5).

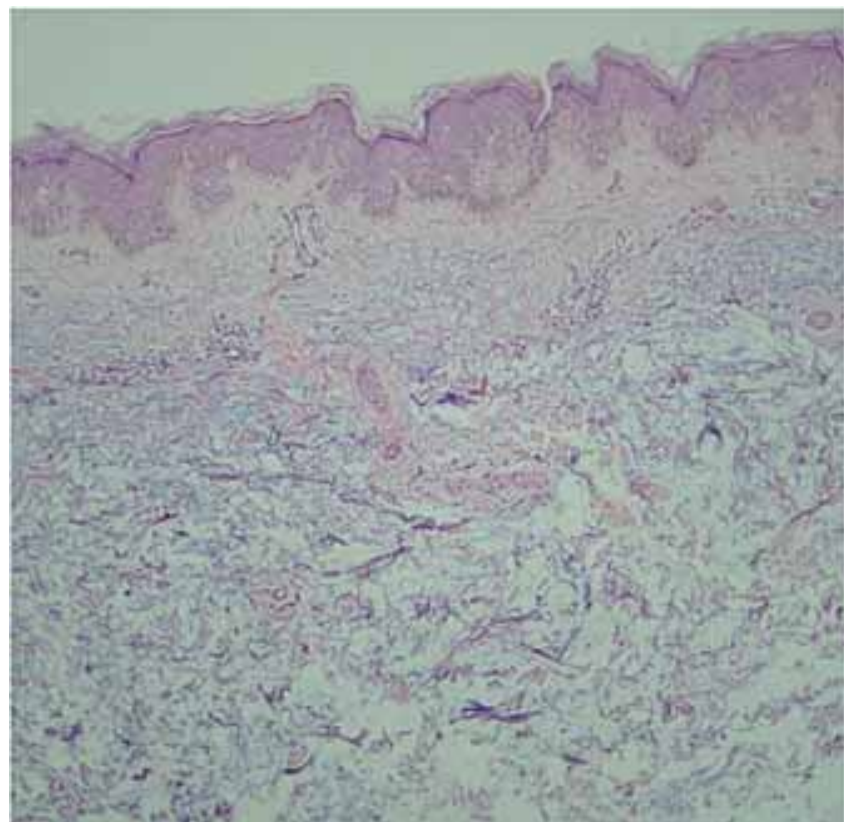

Figure 3: Histopathological examination (H\&E, $x 40)$ showing epidermis and papillary dermis without abnormalities. Accumulation of mucin in reticular dermis

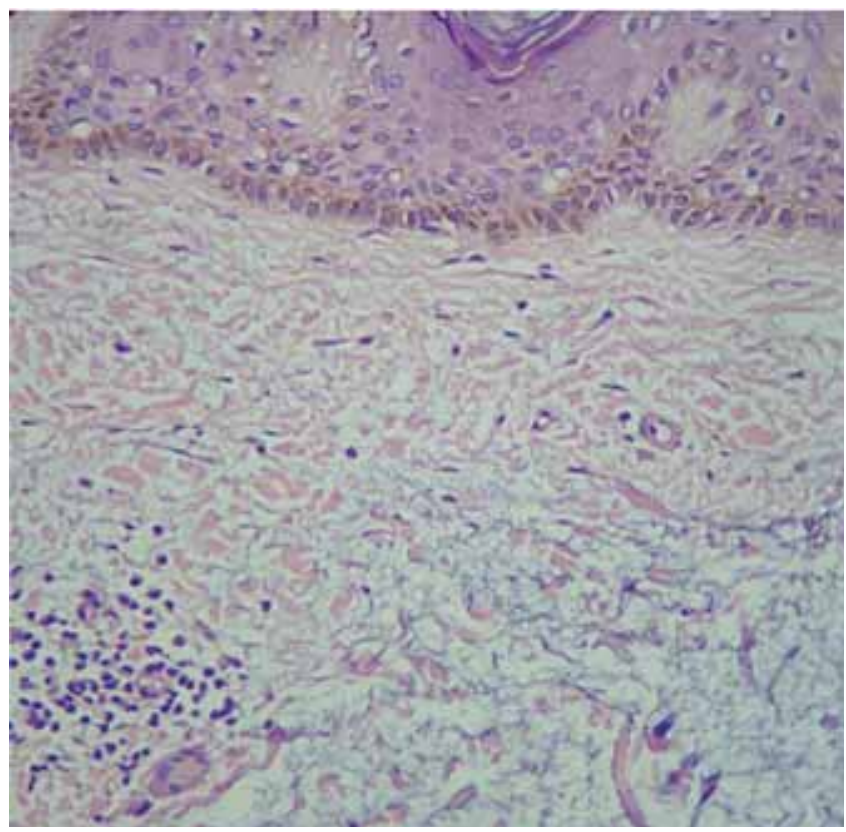

Figure 4: Histopathological examination (H\&E, x100). Accumulation of mucin in reticular dermis causing separation of collagen bundles 


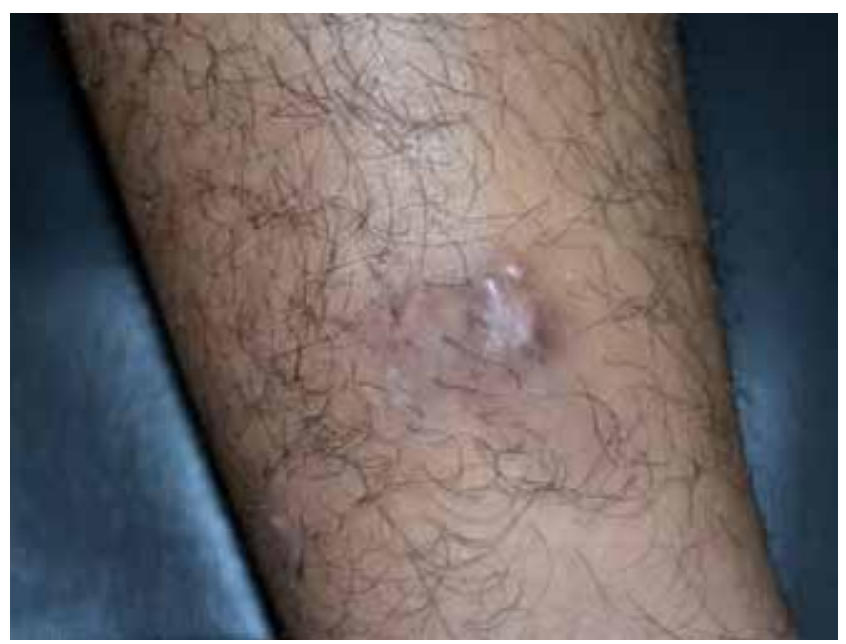

FIGURE 5: Response after 1 application of intralesional corticosteroid

\section{DISAUSSION}

Graves' disease is an autoimmune disease of the thyroid gland which monitors the production of directed autoantibodies mainly against the receptor of thyroid-stimulating hormone (TSH). This is the most common cause of hyperthyroidism, which affects from 15 to 50 per 100.000 people a year, predominantly females. Characteristic clinical findings include exophthalmos, pretibial myxedema and acropathy. These peripheral manifestations of Graves' disease are prone to occur in a chronological order, exophthalmos first (in $30 \%$ of patients), followed by dermopathy (4\% of patients) and then acropathy (less than $1 \%$ of patients). ${ }^{2}$

The clinical forms of pretibial myxedema may vary from one diffuse non-depressible edema, found in the majority of cases, to nodules, plaques, polypoid lesions and manifestations similar to elephantiasis. Al- though the most frequent site is the pretibial region, its presentation may be in any part of the skin, including head, trunk, limbs and under scars. Lesions may vary in color, and may look like "orange peel", due to prominent hair follicles. Typically asymptomatic, they are rarely pruritic or painful. In severe cases, associated with acropathy, osseous pain may result from an underlying periosteal reaction. Hyperhidrosis, hypertrichosis and neuropathies have also been reported. ${ }^{1-3}$

Treatment of pretibial myxedema aims to diminish the production of hyaluronic acid by fibroblasts. Topical steroids with occlusive dressings and compression, intralesional steroids, systemic corticosteroids, pentoxifylline, gamma globulin, plasmapheresis, surgical excision and immunotherapy have been employed with varied success rates. ${ }^{1}$

Many times the lesion remits spontaneously, with the drug treatment used purely for cosmetic interests. Corticoids are the first indication for drug treatment of pretibial myxedema lesions, administered as an occlusive dressing; however, application of intralesional corticoid has already been approved as an efficient therapy, with good results. ${ }^{4-6}$

In order to achieve remission of lesions with topical application it is necessary to apply it as an occlusive dressing every night, during 4 to 6 weeks. ${ }^{1}$ In comparison with corticoid intralesional injection treatments, which present the remission of lesion with a monthly injection, the topical treatment is slower and more laborious for the patient. The adverse effects reported with the use of intralesional corticoids are related with the onset of hyperpigmentation, atrophy and irregularities on the surface of skin. ${ }^{1}$

The responses to the intralesional corticoid vary according to the patient. The conduct was acceptable considering its resolubility and the benign character of lesion. 


\section{REFERENCES}

1. Fatourechi V. Pretibial Myxedema - Pathophysiology and Treatment Options. Am J Clin Dermatol. 2005;6:295-309.

2. Anderson CK, Miller OF 3rd. Triad of exophthalmos, pretibial mydema, and acropachy in a patient with Graves ' disease. J Am Acad Dermatol. 2003;48:970-2.

3. Schwartz KM, Fatourechi V, Ahmed DD, Pond GR. Dermopathy of Graves' Disease(Pretibial Myxedema): Long-Term Outcome. J Clin Endocrinol Metab. 2002:87:438-46.

4. Vannucchi G, Campi I, Covelli D, Forzenigo L, Beck-Peccoz P, Salvi M. Treatment of Pretibial Myxedema with Dexamethazone Injected Subcutaneously by Mesotherapy Needles. Thyroid. 2013;23:626-32.

5. Engin B, Gümüşel M, Ozdemir M, Cakir M. Successful combined pentoxifylline and intralesional triamcinolone acetonide treatment of severe pretibial myxedema. Dermatol Online J. 2007;13:16

6. Deng A, Song D. Multipoint subcutaneous injection of long-acting glucocorticid as a cure for pretibial myxedema. Thyroid. 201;21:83-5.

MAILING ADDRESS:

Luana Oliveira Ramos

Rua A purinã - Praça 14

Centro

69020-170 - M anaus - A M

Brazil

E-mail: oliv.luana@hotmail.com

H ow to cite this article: Ramos LO, Mattos PC, Figueredo GLP, Maia AAA, Romero SAR. Pre-tibial myxedema: treatment with intralesional corticosteroid . An Bras Dermatol. 2015;90 (3 Suppl 1):S143-6. 